Original Research Paper

\title{
Rice Starch as Coating Materials for Microencapsulation of Gamma Oryzanol to Improve its Stability
}

\author{
${ }^{1}$ Anuchita Moongngarm, ${ }^{1}$ Jeerasupa Amornpan, \\ ${ }^{1}$ Amaraphon Homduang, ${ }^{2}$ Cai Haoteng and ${ }^{3}$ Ana Jelekovac \\ ${ }^{1}$ Department of Food Technology and Nutrition, Faculty of Technology, \\ Research Unit of Nutrition and Health, Mahasarakham University, Maha Sarakham, Thailand \\ ${ }^{2}$ Faculty of Food Science, South China Agricultural University, China \\ ${ }^{3}$ Faculty of Food Technology and Biotechnology, University of Zagreb, Croatia
}

\author{
Article history \\ Received: 30-06-2016 \\ Revised: 26-08-2016 \\ Accepted: 26-08-2016 \\ Corresponding Author: \\ Anuchita Moongngarm \\ Department of Food \\ Technology and Nutrition, \\ Faculty of Technology, \\ Research Unit of Nutrition and \\ Health, Mahasarakham \\ University, Maha Sarakham \\ 44150, Maha Sarakham, \\ Thailand \\ Email: anuchitac@yahoo.co.th
}

\begin{abstract}
Gamma Oryzanol (GO) is a phytochemical with the ability to prevent oxidation. GO is normally degraded through oxidation by oxygen and sunlight. Microencapsulation is an important technique used to retard oxidation. This study compared rice starch and Whey Protein Isolate (WPI) as coating materials and investigated the stability of encapsulated GO during storage under activated oxidation conditions. The GO was encapsulated using native rice starch, pregelatinized rice starch, WPI and pregelatinized rice starch combined with WPI (ratio 1:1). The GO concentration, Peroxide Value (PV) and Thiobarbituric Acid Reactive Substance (TBARS) were used to evaluate the effectiveness of the different wall materials. GO encapsulated with pregelatinized rice starch combined with WPI had the greatest stability with the highest GO content $\left(164.54 \mathrm{mg} \mathrm{g}^{-1}\right.$ sample) remaining after 28 days' storage and lowest PV and TBARS values. The result indicated that pregelatinized rice starch used either with or without WPI can be applied as wall material to protect GO from oxidation and improve its stability.
\end{abstract}

Keywords: Gamma Oryzanol, Microencapsulation, Rice Starch, Spray Drying

\section{Introduction}

Gamma Oryzanol ( $\gamma$-oryzanol, GO) is an ester of trans-ferulic acid (trans-hydroxycinnamic acid) and sterol or triterpene alcohols. The chemical structure consists of ferulic acid and sterol or triterpene alcohols such as cycloartenol and 24-methylenecycloartanol connected by ester bonding. GO has been reported to decrease plasma and serum cholesterol (Yoshino et al., 1989; Gerhardt and Gallo, 1998), decrease cholesterol absorption (Seetharamaiah et al., 1990) decrease platelet aggregation (Nakayama et al., 1987) and effectively treat hyperlipidemia (Murase and Lishima, 1963). Its antiinflammatory and anti-tumor was also reported which is related to its antioxidant properties (Juliano et al., 2005). The mechanisms of antioxidant activity are from the hydroxyl group $(\mathrm{OH})$ of the ferulic acid as part of the gamma oryzanol molecule. The ferulic acid can donate hydrogen atoms to free radical and it becomes stable Sterylferulate Radical (SF) and SF radical may also have the ability to break the chain reaction (Kochar, 2000).
Rice bran and rice bran oil are a good source of GO. Crude rice bran oil contains 1.5 to $2.9 \%$ of oryzanol and during refining about $90 \%$ of the oryzanol goes into the unsaponifiable matter as a refinery waste (Gopalakrishna, 2003). It contained as high as $3000 \mathrm{mg} \mathrm{kg}^{-1}$ in unsaponifiable matter obtained from rice bran oil ( $\mathrm{Xu}$ and Godber, 1999).

Usually, GO is degraded by oxidation resulting from oxygen in the air and sunlight. Microencapsulation is an important technique that might protect or delay the oxidation of GO due to it provides several advantages in the food industry by protecting the core materials from their environments. Microencapsulation is defined as a process in which tiny particles or droplets of the core product are surrounded by a coating material, or embedded in a homogeneous or heterogeneous matrix, to give small capsules with many useful properties (Gharsallaoui et al., 2007). This method has been widely applied in the food industry for encapsulating vitamins, minerals and other sensitive ingredients. The 
microcapsules offer the food processors a method to protect sensitive food components (Suh et al., 2007).

Whey protein concentrate, WPI and gelatin have been extensively studied as coating materials in microencapsulation. Carbohydrates such as maltodextrins and corn syrup solids are usually used in the microencapsulation of food ingredients. These compounds are expected to have good microencapsulating properties because they exhibit low viscosities at high solids contents and good solubility (Gharsallaoui et al., 2007). In this study, WPI and rice starch was apply as coating material because WPI is one of the most popular material and rice starch is natural polymer occurring in rice grain the same as GO. However, rice starch still has some limited as it lacks the interfacial properties and its low solubility. Therefore, when used, it might need to modify its structure or used with other encapsulating materials like proteins or gums to improve it solubility. The aim of the study was to investigate the potential of rice starch as a coating material and to evaluate the stability of encapsulated GO stored in activated oxidation conditions.

\section{Materials and Methods}

\section{Materials}

Whey Protein Isolate (WPI), rice starch and Span 80 were purchased from Syntrax Inc. (Mo, USA) and Sigma-Aldrich (Mo, USA), respectively. GO was purchased from Tsuno Food Industrial Co., Ltd. (Wakayama, Japan). All chemicals used in the experiments were of analytical grade.

\section{Preparation of Microencapsulation}

The microencapsulation process followed the method of Lee et al. (2009). Four different coating materials (native rice starch, pregelatinized rice starch, WPI and pregelatinized rice starch combined with WPI (ratio 1:1)) were dissolved in distilled water at $15 \%(\mathrm{w} / \mathrm{w})$ concentrations. Pregelatinized starch was obtained by heating the native starch solution for $10 \mathrm{~min}$ at $90-100^{\circ} \mathrm{C}$. Each coating material solution was then added with $1 \mathrm{~mL}$ of Span 80 and stirred on a magnetic stirrer for $30 \mathrm{~min}$. The core material (GO) was then added to the mixtures in ratio 1:4. The obtained emulsions were then homogenized at 10,000 rpm for another $30 \mathrm{~min}$ using homogenizer (T25, Ika works, Inc., USA). The GO emulsions were dried using a spray drying technique. The $15 \%$ soluble solid solution was maintained under slow agitation during the spray drying process. The spray dryer (Lab Plant SD-04, UK) was operated at an air flow rate of $30 \mathrm{~mL} / \mathrm{min}$, with entrance and exit air temperatures of $170 \pm 2$ and $113 \pm 2^{\circ} \mathrm{C}$, respectively, air pressure $5 \mathrm{kgf} / \mathrm{cm}^{2}$ and aspersion nozzle diameter of $0.7 \mathrm{~mm}$.

\section{Measurement of Microstructure of Encapsulated GO}

The microstructure of the encapsulated GO was measured using Scanning Electron Microscopy (SEM) at a voltage of $10 \mathrm{kV}$.

\section{Determination of $G O$}

The GO content was determined using HPLC, by quantifying the amount of $\mathrm{GO}$ released from the microcapsules. Encapsulated GO was extracted with methanol to final concentration of $1,000 \mu \mathrm{g} \mathrm{mL}$, following the method of (Chen and Bergman, 2005). Analysis of GO was performed using Reversed Phase High Performance Liquid Chromatography (RP-HPLC) following the methods of Moongngarm and Saetung (2010) and Singh and Sigh (2003).

\section{Determination of Cold Water Solubility}

Cold Water Solubility (CWS) of each coating core material substance and obtained powders was determined following the method of Singh and Sigh (2003):

$$
C W S \%=\frac{\text { grams of solid in supernatant } \times 10}{\text { grams of sample }} \times 100
$$

\section{Stability of Encapsulated GO}

The encapsulated GO in the three types of wall material was exposed to heat-induced oxidation by incubating at $60 \pm 1^{\circ} \mathrm{C}$ in an incubator for 28 days in polyethylene bags in the light. The samples were taken out every 7 days to determine the remaining GO, peroxide value (Mielnik et al., 2003) and Thiobarbituric Acid Reactive Substance (TBARS) (Mukerjea et al., 2007).

\section{Statistical Analysis}

The means and standard deviations of GO content, peroxide and TBARS values were reported in triplicate for each sample. Multiple comparison tests were performed using Duncan's multiple range tests to determine the significant difference between treatments. Statistical significance was stated at $P<0.05$.

\section{Results}

\section{Microstructure of Encapsulated GO}

The morphological properties of the core material and three types of coating materials were observed by SEM. Figure 1 demonstrates the scanning electron micrographs of pure core material (GO) (A1, A2), GO encapsulated with native rice starch $(\mathrm{B} 1, \mathrm{~B} 2), \mathrm{GO}$ encapsulated with pregelatinized rice starch $(\mathrm{C} 1, \mathrm{C} 2)$ and GO encapsulated with WPI (D1, D2) and GO encapsulated with the combination of pregelatinized rice starch and WPI (E1, E2). 

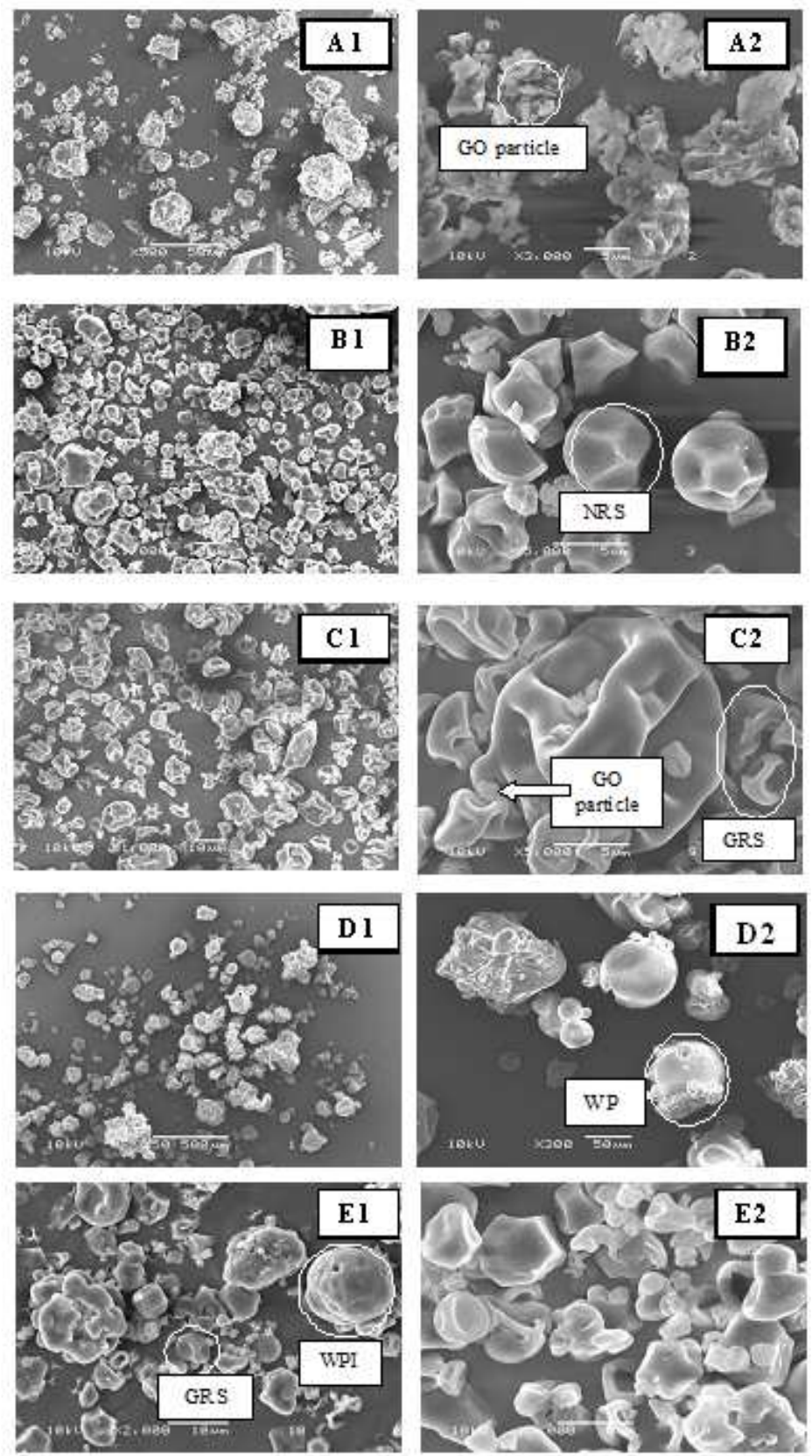

Fig. 1. Scanning electron micrographs of microcapsules, A1, A2 = unencapsulated GO; B1, B2 = GO encapsulated with native rice starch; C1, C2 = GO encapsulated with pregelatinized rice starch; D1, D2 = GO encapsulated with WPI; E1, E2 = GO encapsulated with a combination of pregelatinized rice starch and WPI; NRS = native rice starch granule; GRS = pregelatinized rice starch granule; WP = WPI particle with GO particle; WPI = whey protein isolate

Effect of Coating Material on the Solubility of Encapsulated GO

The CWS of each encapsulated GO sample is indicated in Fig. 2. GO encapsulated with WPI and WPI with pregelatinized rice starch were significantly highest at 95.56 and $93.41 \%$ respectively, third highest was GO encapsulated with pregelatinized rice starch at $89.64 \%$ and the two remaining samples displayed significantly lower values of CWS.

Effect of Microencapsulation on the Stability of Encapsulated $G O$

The remaining level of $\mathrm{GO}$ coated with the different materials after storage at $60^{\circ} \mathrm{C}$ for 28 days is shown in Fig. $3 \mathrm{~A}$ and $\mathrm{B}$. The results showed that coating materials 
improved the stability of GO. The GO levels on the first day for coated samples ranged from 215.62 to $219.52 \mathrm{mg}$ $\mathrm{g}^{-1}$, with the concentration of GO in the unencapsulated sample at $742.33 \mathrm{mg} \mathrm{g}^{-1}$ as the concentration from the supplier. The GO reduced significantly after storage for 7 , 14 and 21 days, with the lowest levels after 28 days. The highest decreasing rate was found in unencapsulated GO (96.51\%, Fig. 3B) whereas the maximum amount of GO remaining after 28 days was recorded in pregelatinized rice starch combined with WPI at $164.54 \mathrm{mg} \mathrm{g}^{-1}$.
Peroxide values of GO encapsulated with different coating materials are shown in Fig. 4. The types of coating materials significantly affected the peroxide value after 7 days of storage until 28 days $(P<0.05)$. GO unencapsulated had the highest peroxide value after 7 days until 28 days storage at $34.63 \mathrm{meq} \mathrm{kg} \mathrm{kg}^{-1}$ $(P<0.05)$, while GO coated by pregelatinized rice starch and pregelatinized rice starch combined with WPI had the lowest peroxide value at the end of storage $\left(16.74 \mathrm{meq} \mathrm{kg}^{-1}\right)(P<0.05)$.

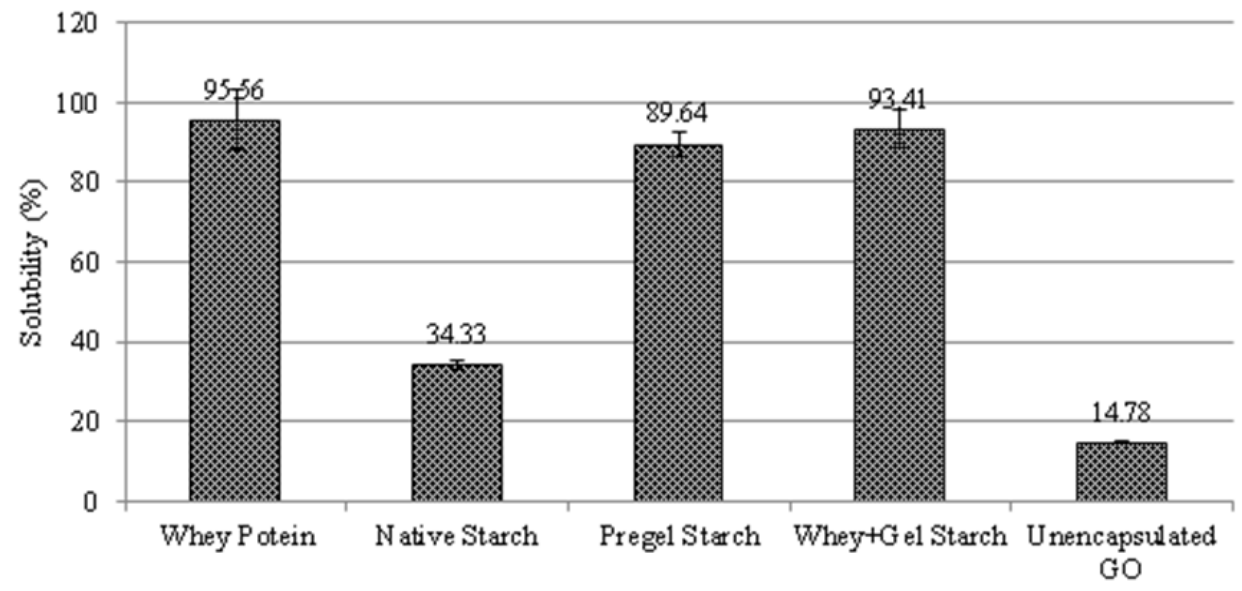

Fig. 2. Effect of coating materials on cold water solubility of encapsulated GO

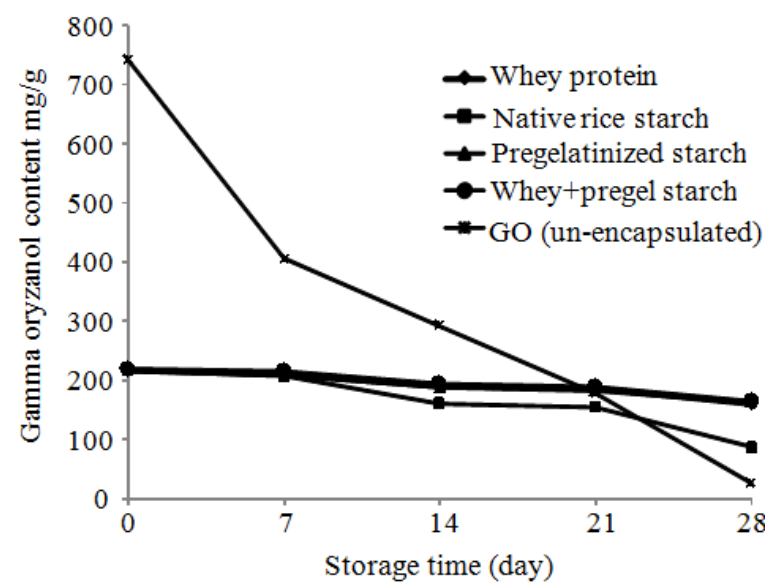

(A)

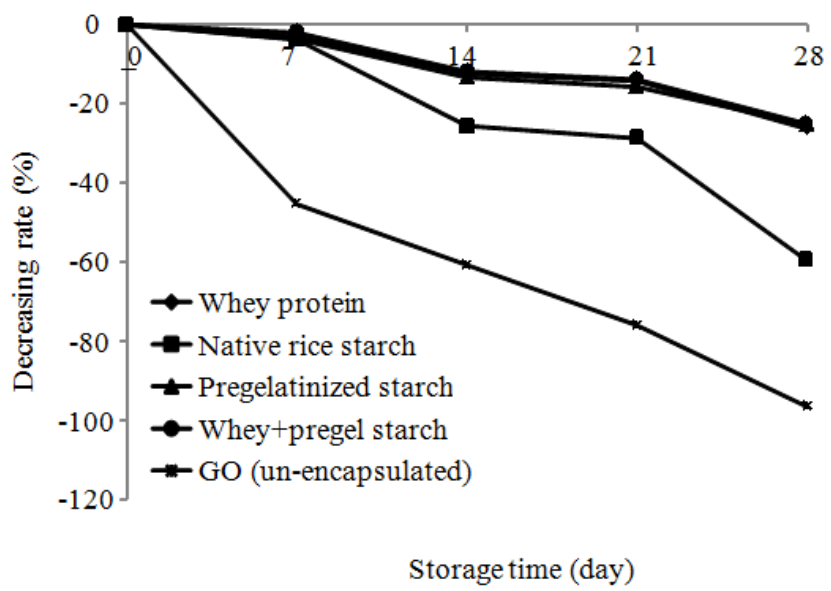

(B)

Fig. 3. Change of $\gamma$-oryzanol encapsulated with different coating materials; GO remaining during storage (A); decreasing rate\% (B)

\section{Effect of Microencapsulation on TBARS Value of Encapsulated $\mathrm{GO}$}

The concentration of malondialdehyde was obtained from the reaction with thiobarbituric acid which produces a red product which absorbs light at wavelength $532 \mathrm{~nm}$ and has molar absorptivity at 27.5 absorbance units/ $\mu \mathrm{mol}$ (Suh et al., 2007). TBARS values of GO encapsulated with different coating materials are shown in Fig. 5. The results indicated that coating materials affected the amount of malondialdehyde at 7 days of storage until the end of storage at 28 days $(P<0.05)$. The unencapsulated GO had the highest TBARS value at the end of storage at $3.09 \mathrm{mg} \mathrm{kg}^{-1}$, while the GO coated by pregelatinized rice starch and pregelatinized rice starch combined with WPI were more stable until 28 days of storage with the TBARS of 2.41 and $2.36 \mathrm{mg} \mathrm{kg}^{-1}$, respectively. 


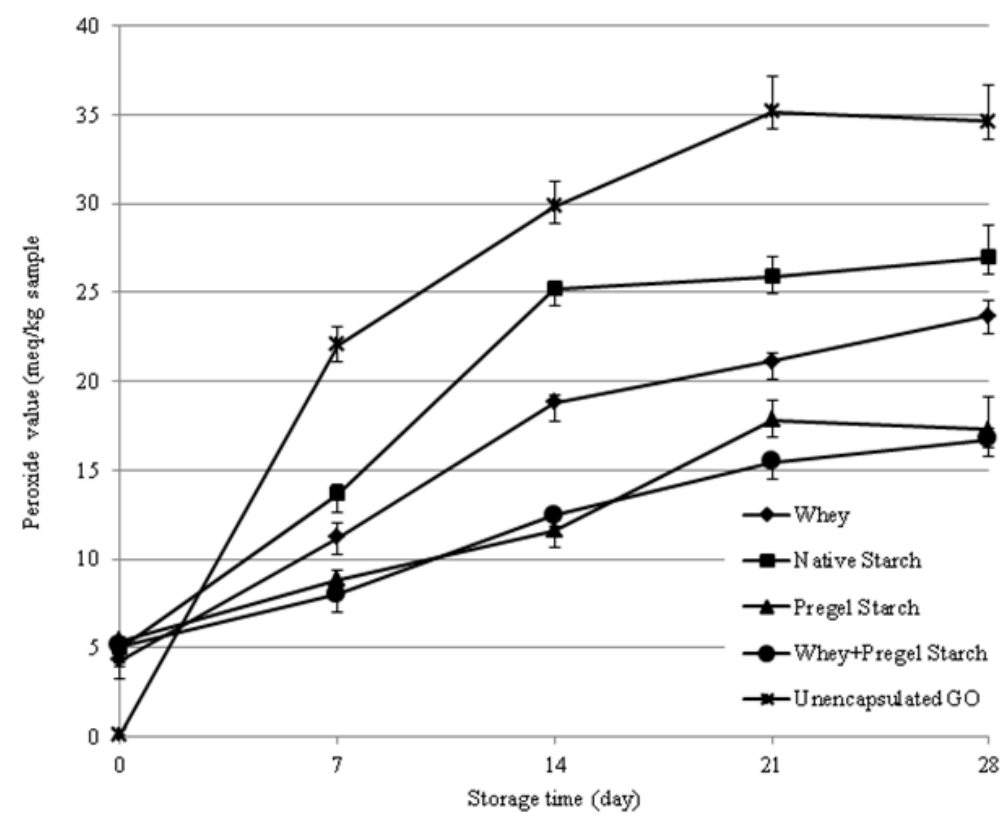

Fig. 4. Peroxide value change of encapsulated GO

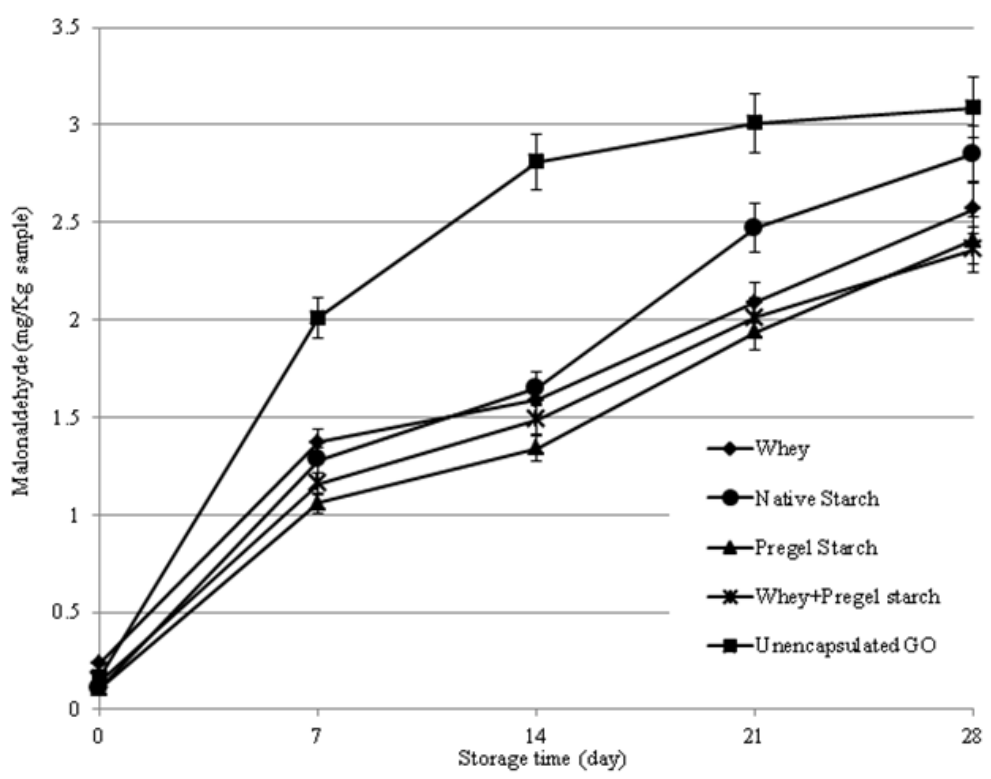

Fig. 5. TBARS value change of encapsulated $\gamma$-oryzanol

\section{Discussion}

The morphological characteristics of the core material (GO) and encapsulated GO showed that all microencapsulation types illustrated inhomogeneous microcapsules depending on the structure of the coating materials. The core material was surrounded by coating materials. The native starch granule show mainly intact polygonal shapes whereas those of pregelatinized starch showed irregular shape with shrink appearance as affected by heating.
The solubility of all samples was significantly differed from each other, except that of WPI and pregelatinized rice starch combined with WPI. Low solubility of GO encapsulated with native rice starch and unencapsulated GO was to be expected when considering its chemical composition. Starch is composed of amylose and amylopectin which combine together as a water-insoluble granule. The secondary and tertiary structures hold the molecules together and give the insoluble character to the starch granules (Decker and Hultin, 1992). The 
gelatinization of starch at high temperature, before spray drying, could improve the solubility of rice starch indicating by the higher water solubility than that of the GO encapsulated using native rice starch. Higher values of CWS for GO micro-encapsulated with a combination of pregelatinized starch and WPI might be due to the surface activity properties of WPI allowing them to stabilize in water.

In the study on the stability of GO, the concentration of $\mathrm{GO}$ remaining during storage was measured as an indicator of GO stability. The GO reduced significantly during storage in high temperature $\left(60^{\circ} \mathrm{C}\right)$ which the lowest level was observed at 28 days of storage. The reduction of Go might be due to the degradation of the coating material at high temperature (Choe and Min, 2007) which resulted in oxidation of GO and the instability of GO during storage.

The PV and TBARS values were used as the oxidation index to evaluate the effect of microencapsulation on protecting the GO from oxidation. The PV measures the extent of the primary oxidation of fats and oils (Pokorny et al., 2001). The results suggested that microencapsulation of GO could prevent oxidation by limiting the diffusion of oxygen into the matrix of GO. The coating material also protected the oil and related compounds such as GO from oxidation by immobilizing the free radicals. When the storage time increased, the peroxide value increased. The results were similar to those reported by Choe and Min (2007). However, the peroxide compounds were unstable at high temperatures and broke into other compounds such as aldehydes, ketones, esters, alcohols and short-chain hydrocarbons which can be evaluated through the TBARS value. The coating materials could also reduce the level of malondialdehyde shown by the low level of TBARS values of encapsulated GO. The malondialdehyde was produced from the GO oxidation which the formation of malondialdehyde of encapsulated GO increased slower than that of unencapsulated GO. These results were in agreement with the study of Pourashouri et al. (2014) who investigated the oxidative stability of spray-dried microencapsulated fish oils with different wall materials. The values of TBARS in this study were higher than the values reported by Qazi et al. (2015). This might be due to the storage rage temperature of this study was higher $\left(60^{\circ} \mathrm{C}\right)$ than those of Qazi et al. (2015) stored sample in 4 and $25^{\circ} \mathrm{C}$.

\section{Conclusion}

Different kinds of coating materials had different effect on the stability of GO. The GO encapsulated with pregelatinized rice starch and pregelatinized rice starch with WPI showed the highest amount of GO content remaining and lowest PV and TBARS value. The study suggested that rice starch has the potential for use as a coating material for encapsulating and protecting GO from oxidation. However, further studies with modified emulsion compositions and microencapsulation conditions are needed to achieve higher micro-encapsulation efficiency.

\section{Acknowledgement}

The study was financially supported by Mahasarakham University, Thailand, grant number 2558A10902132.

\section{Funding Information}

Mahasarakham University (NRCT, Fiscal year 2015), Mahasarakham, Thailand

\section{Author's Contributions}

All authors of this research article have directly participated in the planning, execution and analysis of this study.

\section{Ethics}

The contents of this manuscript will not be copyrighted, submitted, or published elsewhere. There are no directly related manuscripts, published or unpublished, by any authors of this study. My Institute's, Mahasarakham University, representative, is fully aware of this submission.

\section{References}

Chen, M.H. and C.J. Bergman, 2005. A rapid procedure for analyzing rice bran tocopherol, tocotrienols and $\gamma$-oryzanol contents. J. Food Comp. Anal., 18: 139-151. DOI: 10.1016/j.jfca.2003.09.004

Choe, E. and D.B. Min, 2007. Chemistry of deep-fat frying oils. J. Food Sci., 72: 77-86. DOI: $10.1111 /$ j.1750-3841.2007.00352.x

Decker, E.A. and H.O. Hultin, 1992. Lipid Oxidation in Muscle Food Via Redox Iron, 33-54, Inst. In: Lipid Oxidation in Food, Angelo, A.J. (Ed.), ACS Press, New York, ISBN-10: 0841224617, pp: 33-54.

Gerhardt, A.L. and N.B. Gallo, 1998. Full-fat rice bran and oat bran similarly reduce hypercholesterolemia in humans. J. Nutr., 128: 865-869. PMID: 9566995

Gharsallaoui, A., G. Roudaut, O. Chambin, A. Voilley and R. Saurel, 2007. Applications of spray-drying in microencapsulation of food in-gredients: An overview. Food Res. Int., 40: 1107-1121. DOI: 10.1016/j.foodres.2007.07.004

Gopalakrishna, A.G., 2003. Value-added products from rice bran oil industry. SAARC.

Juliano, C., M. Cossu, M.C. Alamanni and L. Piu, 2005. Antioxidant activity of gamma-oryzanol: Mechanism of action and its effect on oxidative stability of pharmaceutical oils. Int. J. Pharm., 299: 146-154. DOI: 10.1016/j.ijpharm.2005.05.018 
Kochar, S.P., 2000. Stabilization of frying oils with natural antioxidant compounds. Eur. J. Lipid Sci. Technol., 102: 552-559.

Lee, S.W., S.Y. Kang and S.H. Han, 2009. Influence of modification method and starch concentration on the stability and physical properties of modified potato starch as wall materials. Eur. Food Res. Technol., 228: 449-455. DOI: 10.1007/s00217-008-0952-5

Mielnik, M.B., K. Aaby and G. Skrede, 2003. Commercial antioxidants control lipid oxidation in mechanically deboned turkey meat. Meat Sci., 65: 1147-1155. PMID: 22063697

Moongngarm, A. and N. Saetung, 2010. Comparison of chemical compositions and bioactive compounds of germinated rough rice and brown rice. Food Chem., 122: 782-788. DOI: $10.1016 /$ j.foodchem.2010.03.053

Mukerjea, R., G. Slocum and J.F. Robyt, 2007. Determination of the maximum water solubility of eight native starches and the solubility of their acidicmethanol and -ethanol modified analogues. Carbohydr. Res., 342: 103-110. DOI: 10.1016/j.carres.2006.10.022

Murase, Y. and H. Lishima, 1963. Clinical studies of oral administration of gamma-oryzanol on climacteric complaints and its syndrome. Obstet. Gynecol. Prac., 12: 147-149.

Nakayama, S., A. Manabe, J. Suxuki, K. Sakamoto and T. Inagaki, 1987. Comparative effects of two forms of gamma-oryzanol in different sterol compositions on hyperlipidemia induced by cholesterol diet in rats. Japan J. Pharmacol., 44: 135-143. PMID: 3656772

Pokorny, J., N. Yanishlieva and M. Gordon, 2001. Antioxidants in Food: Practical Applications. 1st Edn., CRC Press, New York, ISBN-10: 0849312221, pp: 288.
Pourashouri, P., B. Shabanpour, S.H. Razavi, S.M. Jafari and A. Shabani et al., 2014. Oxidative stability of spray-dried microencapsulated fish oils with different wall materials. J. Aquatic Food Product Technol., 23: 567-578.

DOI: $10.1080 / 10498850.2012 .738357$

Qazi, H.J., H. Majeed, W. Safdar, J. Antoniou and Z. Fang, 2015. A novel approach for microencapsulation of nanoemulsions to overcome the oxidation of bioactives in aqueous phase. Adv. J. Food Sci. Technol., 7: 388-394.

Seetharamaiah, G.S., T.P. Krishnakantha and N. Chandrasekhara, 1990. Influence of oryzanol on platelet aggregation in rats. J. Nutr. Sci. Vitaminol., 36: 291-297. DOI: 10.3177/jnsv.36.291

Singh, N. and J. Sigh, 2003. Studies on the morphological and rheological properties of granular cold water soluble corn and potato starches. Food Hydrocoll., 17: 63-72. DOI: 10.1016/S0268-005X(02)00036-X

Suh, M.H., S.H. Yoo and H.G. Lee, 2007. Antioxidative activity and structural stability of microencapsulated $\gamma$-oryzanol in heat-treated lards. Food Chem., 100: 1065-1070. DOI: 10.1016/j.foodchem.2005.11.013

$\mathrm{Xu}, \mathrm{Z}$. and J.S. Godber, 1999. Purification and identification of components of $\gamma$-oryzanol in rice bran oil. J. Agric. Food Chem., 47: 2724-2728. DOI: $10.1021 / \mathrm{j} f 981175 \mathrm{j}$

Yoshino, G., T. Kazumi, M. Amano, M. Tateiwa and T. Yamasaki et al., 1989. Effects of gammaoryzanol on hyperlipidemic subjects. Curr. Ther. Res., 45: 543-552. 